\title{
Infrared and visual lunar occultations measurements of stellar diameters and new binary stars detections at the Calar Alto $1.5 \mathrm{~m}$ telescope ${ }^{\star}$
}

\author{
O. Fors ${ }^{1,2}$, A. Richichi ${ }^{3}$, J. Núñez ${ }^{1,2}$, and A. Prades ${ }^{4}$ \\ 1 Departament d'Astronomia i Meteorologia, Universitat de Barcelona, Av. Diagonal 647, 08028 Barcelona, Spain \\ 2 Observatori Fabra, Camí de l'Observatori s/n, 08035 Barcelona, Spain \\ ${ }^{3}$ European Southern Observatory, Karl-Schwarzschild-Str. 2, 85748 Garching bei München, Germany \\ ${ }^{4}$ Escola Universitária Politécnica de Barcelona, Universitat Politècnica de Catalunya, 08028 Barcelona, Spain
}

Received 16 October 2003 / Accepted 9 February 2004

\begin{abstract}
We present a program of routine lunar occultations, at optical and near-IR wavelengths, recently started at the $1.5 \mathrm{~m}$ Spanish telescope at the Calar Alto Observatory. Both a CCD and an infrared array detector are used. The program is aimed mainly at the detection and investigation of binary systems, although results in other areas of stellar research are also anticipated. Occultations are reported for a total of 40 stars. Among these, SAO 164567, SAO 78258 and AG+24 788 have been discovered to be binaries, with projected separations as small as 0.'006. Furthermore, binarity is suspected in the case of SAO 78119 and SAO 79251. Additionally, the angular diameter of the late-type giant 30 Psc and of the infrared star V349 Gem have been accurately measured, the latter for the first time. We finally evaluate the instrumentation performance in terms of the limiting magnitude and angular resolution, and discuss applications to larger telescopes.
\end{abstract}

Key words. astrometry - occultations - stars: binaries: close - stars: binaries: visual - instrumentation: detectors

\section{Introduction}

The technique of lunar occultations (LO) has established itself as a very simple and powerful means to achieve high angular resolution. It consists essentially in performing highspeed photometry of the light curve produced when a background star is occulted by the limb of the Moon. Angular resolution information on the occulted source on the scale of one milliarcsecond (mas) can be retrieved from the analysis of the diffraction fringes, either through model-dependent or model-independent approaches (Richichi 1989). Since the occultation is a diffraction phenomenon produced at the lunar limb rather than in the telescope, these observations are characterized by several properties which are significantly different from the ones typically encountered with other techniques for high angular resolution such as adaptive optics, speckle or long-baseline interferometry. For example, the limiting angular resolution is not set by the size of the telescope nor by the wavelength of observation, at least to a first approximation. Also, it is not directly influenced by the quality of the seeing.

While these properties make the LO technique very attractive, a number of shortcomings limit its application

\footnotetext{
Send offprint requests to: O. Fors, e-mail: ofors@am.ub.es

$\star$ Appendix A is only available in electronic form at http://www. edpsciences.org
}

considerably. First and foremost, occultations can be observed only for sources which lie on the apparent orbit of the Moon. This restricts the candidates to a narrow belt around the Zodiac, covering approximately $10 \%$ of the celestial sphere. Second, LO are fixed-time events, which need careful planning and the successful combination of technical and meteorological readiness. Finally, each LO event only provides a one-dimensional scan of the source, along a direction which is determined by the lunar motion and the source position. Depending on the conditions, a given source can be occulted only once or several times over a period of a few months or very few years. In this case, some limited two-dimensional information can be obtained.

The combination of these advantages and limitation, makes the LO technique particularly appealing especially for small and medium-sized telescopes, where a routine program of observations can be established. The availability of relatively cheap detectors for visual and near-infrared (NIR) fast photometry is bringing this method within the budget of most observatories. In this respect, we note the benefits of commercial, relatively cheap CCDs developed for amateur astronomers on one side, and previous-generation NIR arrays of small format on the other side. In their respective wavelenght ranges, both kinds of detector offer a quality sufficient for the purpose of LO observations, where the noise is essentially set by the lunar background. 
These considerations have motivated us to start an LO program on the $1.5 \mathrm{~m}$ Spanish telescope of the Calar Alto Observatory. This program is independent of previous similar efforts at the same observatory, in which some of us were involved. Unfortunately, those efforts are presently stalled, given the difficulty of allocating time to the required instrumentation. The present program is carried on at a different telescope and with different instrumentation. In addition to the allocation of sufficient observing time, the program is becoming a success thanks to the availability of two instruments for which specific adaptations allow a rapid sampling of the lightcurves, as described in Sect. 2. In Sect. 3 we describe the first results obtained on a number of sources, including new binary detections and stellar angular diameters. In Sect. 4, we evaluate the performance of the two instruments employed, and extrapolate their possible application to larger telescopes.

\section{Observations and data reduction}

Visual and NIR observations were carried out with the $1.5 \mathrm{~m}$ telescope of the Observatorio Astronómico Nacional in Calar Alto (Spain) during October 2001 and February 2002, respectively. For the visual occultations we employed a Texas Instruments TC-211 CCD, which is assembled as the guiding chip of the SBIG-ST8 camera. This was operated in fast driftscanning mode as described by Fors et al. (2001), allowing us to sample the occultation lightcurves at millisecond rates. For the NIR observations, we made use of the MAGIC camera (Herbst et al. 1993), operated in fast subarray mode with a window size of $8 \times 8$ pixels. This size was found to provide a good compromise between a sufficiently fast sampling rate (typically $8.5 \mathrm{~ms}$ with an integration time of $3 \mathrm{~ms}$ ) and a robust estimation of the background level around the stellar image.

Table 1 lists the parameters of the observed occultations, following closely the format established in Richichi et al. (2002), and previous papers of that series. Columns 1 through 3 list the source identification, the date of the event and the configuration used. The code CA refers to observations with the $\mathrm{CCD}$, and the code $\mathrm{CB}$ to observations with MAGIC. Broad band $R(641 \pm 58 \mathrm{~nm})$ and $K(2.2 \pm 0.4 \mu \mathrm{m})$ filters were used, respectively. Column 4 lists the field of view set either by the diaphragm aperture or by the array subwindow. Columns 5 and 6 list the sampling time of the lightcurves, and the integration time for each data point. Columns 7 through 9 list the total magnitude of the star in the $V, R$ and $K$ filters. The $V$ magnitudes are taken from the literature. In principle, photometric information could be extracted directly from the LO data. However, we lacked an accurate instrument calibration, and we wished to avoid possible systematic biases: this is the case for the CCD drift-scanning technique, since the object does not effectively fit within the single column which is read out at each integration time. Instead, we have collected the $R$ and $K$ magnitudes from the USNO-B1.0 and 2MASS catalogues, respectively. Note that many of the CB sources have $K$ magnitudes above the saturation limit of 2MASS. Although this has been accounted for in the catalogue, systematic errors might still be present and possible examples are discussed in Sect. 4.1. In Col. 10 we report the spectral types, again extracted when available from the literature; in the case of multiple determinations, the most frequent or most recent was used. Finally, Col. 11 lists the distances based on Hipparcos parallaxes, when available. Those values affected by a large uncertainty $(>10 \%)$ have been omitted.

The data were analyzed with the same methods used by Richichi et al. (2002, 2003), and other papers in those series. In particular, a model-dependent least squares method was employed, which uses as free parameters the angular diameter for single stars, and additionally for binary stars the angular diameter of the companion, the projected separation and the brightness ratio. Other free parameters include the rate of the event, the intensity of the background and its time drift. Spurious frequencies due to pick-up of mains power and other effects have been occasionally noticed, and have been digitally filtered. Our data analysis can also include a fit and removal of relatively slow, random fluctuations of the background (due to thin cirrus and lunar halo) and of the stellar intensity (due to image motion and scintillation), by means of modelling through Legendre polynomials as described in the above mentioned papers.

We also used a model-independent method (CAL, Richichi 1989), which is particularly suited for detecting companions at very small separations and the presence of extended circumstellar emission.

\section{Results}

The stars for which a positive result was obtained are listed in Table 2. In the table, the format follows the same style as in Richichi et al. (2002) and other papers of that series. A detailed discussion of the listed quantities is given in Richichi et al. (1996). In summary, the columns list the absolute value of the fitted linear rate of the event $V$ in $\mathrm{m} \mathrm{ms}^{-1}$, its deviation from the predicted rate $V_{\mathrm{t}}$ as computed by us, the local lunar limb slope $\psi$, the true position and contact angles, and the signal-tonoise ratio $(S N R)$. For binary detections, the projected separation and the brightness ratio are given, while for the single stars the angular diameter $\phi_{\mathrm{UD}}$ is reported, under the assumption of a uniform stellar disc. Only in the case of SAO 164567 was the predicted contact angle sufficiently close to zero that even a small difference of $3 \%$ between predicted and measured rate results in an imaginary value of the limb slope. In this case, we list the predicted, rather than measured, PA and CA values.

\section{1. $S A O 164567$}

This star was included in observations both by the Hipparcos satellite, and for radial velocity measurements (Moore \& Paddock 1950; Duflot et al. 1995), but it was never reported as binary.

\section{2. $30 \mathrm{PsC}$}

This long period variable is classified as an oxygen-rich AGB MIII giant without dust emission (Sloan \& Price 1998). It has been catalogued by Hipparcos as suspected nonsingle. One speckle observation with a limiting resolution of 0.'054 was inconclusive in this respect (Mason et al. 1999). 
Table 1. List of the occultation events and of the circumstances of their observation.

\begin{tabular}{|c|c|c|c|c|c|c|c|c|c|c|}
\hline (1) & $(2)$ & (3) & (4) & (5) & (6) & (7) & (8) & (9) & (10) & (11) \\
\hline Source & Date & Tel.+ & $D$ & $\Delta t$ & $\tau$ & $V$ & $R$ & $K$ & Sp. & Dist. \\
\hline & UT & detector & " & $\mathrm{ms}$ & $\mathrm{ms}$ & mag & mag & mag & & $\mathrm{pc}$ \\
\hline SAO 187645 & $22-10-01$ & $\mathrm{CA}$ & 8 & 2.2 & 2.2 & 8.3 & 7.4 & & K1/K2III & \\
\hline SAO 187660 & $22-10-01$ & $\mathrm{CA}$ & 11 & 2.2 & 2.2 & 7.3 & 6.5 & & K2III & 128 \\
\hline SAO 189746 & $24-10-01$ & $\mathrm{CA}$ & 6 & 1.8 & 1.8 & 8.6 & 8.0 & & G8III & \\
\hline SAO 189774 & $24-10-01$ & $\mathrm{CA}$ & 8 & 5.0 & 5.0 & 9.5 & 8.8 & & K1/K2III & \\
\hline SAO 164553 & $25-10-01$ & $\mathrm{CA}$ & 7 & 5.0 & 5.0 & 8.5 & 8.3 & & F0III/IV & \\
\hline SAO 164567 & $25-10-01$ & $\mathrm{CA}$ & 8 & 1.8 & 1.8 & 7.4 & 6.5 & & K5III & \\
\hline SAO 165121 & $26-10-01$ & $\mathrm{CA}$ & 7 & 3.0 & 3.0 & 9.2 & 8.5 & & K1/K2III & \\
\hline SAO 165128 & $26-10-01$ & $\mathrm{CA}$ & 7 & 6.0 & 6.0 & 9.5 & 9.0 & & G2/G3V & \\
\hline SAO 165136 & $26-10-01$ & $\mathrm{CA}$ & 6 & 2.0 & 2.0 & 7.8 & 7.0 & & KOIII & 230 \\
\hline SAO 165578 & $27-10-01$ & $\mathrm{CA}$ & 6 & 2.1 & 2.1 & 6.1 & 5.3 & & K5III & 256 \\
\hline SAO 147033 & $28-10-01$ & $\mathrm{CA}$ & 7 & 1.6 & 1.6 & 7.7 & 6.9 & & K0 & 238 \\
\hline SAO 147032 & $28-10-01$ & $\mathrm{CA}$ & 7 & 1.5 & 1.5 & 7.8 & 7.5 & & F5 & 221 \\
\hline $30 \mathrm{Psc}$ & $28-10-01$ & $\mathrm{CA}$ & 6 & 1.5 & 1.5 & 4.4 & 3.6 & & M3III & 127 \\
\hline SAO 78001 & $22-02-02$ & $\mathrm{CB}$ & 7 & 8.5 & 3.0 & 9.1 & & 7.7 & F0 & \\
\hline SAO 78119 & $22-02-02$ & $\mathrm{CB}$ & 7 & 8.7 & 3.0 & 8.1 & & 4.9 & K0 & \\
\hline SAO 78122 & $22-02-02$ & $\mathrm{CB}$ & 7 & 8.4 & 3.0 & 7.9 & & 5.7 & G5 & 217 \\
\hline SAO 78168 & $22-02-02$ & $\mathrm{CB}$ & 7 & 8.4 & 3.0 & 6.1 & & 3.9 & G8III & 134 \\
\hline SAO 78176 & $22-02-02$ & $\mathrm{CB}$ & 7 & 8.4 & 3.0 & 6.3 & & 4.9 & B3Ib & \\
\hline SAO 78192 & $23-02-02$ & $\mathrm{CB}$ & 7 & 8.4 & 3.0 & 8.4 & & 3.6 & М... & \\
\hline SAO 78197 & $23-02-02$ & $\mathrm{CB}$ & 7 & 8.6 & 3.0 & 8.2 & & 5.3 & K0 & \\
\hline DO 12097 & $23-02-02$ & $\mathrm{CB}$ & 7 & 8.4 & 3.0 & 9.3 & & 5.3 & & \\
\hline SAO 78210 & $23-02-02$ & $\mathrm{CB}$ & 7 & 8.5 & 3.0 & 6.6 & & 4.5 & G5 & 242 \\
\hline V349 Gem & $23-02-02$ & $\mathrm{CB}$ & 7 & 8.3 & 3.0 & 12.2 & & 4.1 & & \\
\hline SAO 78258 & $23-02-02$ & $\mathrm{CB}$ & 7 & 8.5 & 3.0 & 8.2 & & 6.9 & G0 & 198 \\
\hline SAO 78272 & $23-02-02$ & $\mathrm{CB}$ & 7 & 8.5 & 3.0 & 7.3 & & 5.0 & K0 & \\
\hline SAO 79133 & $23-02-02$ & $\mathrm{CB}$ & 7 & 8.5 & 3.0 & 7.9 & & 6.8 & F5 & 72 \\
\hline $\mathrm{AG}+24788$ & $23-02-02$ & $\mathrm{CB}$ & 7 & 8.4 & 3.0 & 10.3 & & 6.4 & K0 & \\
\hline SAO 79162 & $23-02-02$ & $\mathrm{CB}$ & 7 & 8.5 & 3.0 & 5.9 & & 4.8 & F5III-IV & 107 \\
\hline SAO 79176 & $23-02-02$ & $\mathrm{CB}$ & 7 & 8.5 & 3.0 & 9.2 & & 7.6 & G5 & \\
\hline SAO 79194 & $23-02-02$ & $\mathrm{CB}$ & 7 & 8.5 & 3.0 & 8.7 & & 7.5 & F5 & \\
\hline SAO 79214 & $23-02-02$ & $\mathrm{CB}$ & 7 & 8.5 & 3.0 & 7.9 & & 5.6 & G5 & 236 \\
\hline SAO 79236 & $23-02-02$ & $\mathrm{CB}$ & 7 & 8.5 & 3.0 & 8.1 & & 7.0 & F8 & 40 \\
\hline SAO 79251 & $23-02-02$ & $\mathrm{CB}$ & 7 & 8.5 & 3.0 & 8.7 & & 6.3 & K0 & \\
\hline SAO 79257 & $23-02-02$ & $\mathrm{CB}$ & 7 & 8.5 & 3.0 & 8.4 & & 7.4 & F5 & 167 \\
\hline $\mathrm{AG}+24824$ & $23-02-02$ & $\mathrm{CB}$ & 7 & 8.5 & 3.0 & 10.2 & & 7.9 & G0 & \\
\hline$A G+23808$ & $24-02-02$ & $\mathrm{CB}$ & 7 & 8.4 & 3.0 & 10.2 & & 7.8 & K0 & \\
\hline SAO 79302 & $24-02-02$ & $\mathrm{CB}$ & 7 & 8.5 & 3.0 & 8.3 & & 7.9 & $\mathrm{~A} 2$ & 280 \\
\hline SAO 79325 & $24-02-02$ & $\mathrm{CB}$ & 7 & 8.5 & 3.0 & 9.5 & & 7.0 & $\mathrm{~K} 2$ & \\
\hline SAO 79365 & $24-02-02$ & $\mathrm{CB}$ & 7 & 8.5 & 3.0 & 9.3 & & 6.2 & K7 & \\
\hline IRAS $07231+2349$ & $24-02-02$ & $\mathrm{CB}$ & 7 & 8.4 & 3.0 & & & 4.0 & & \\
\hline
\end{tabular}

Two interferometric observations in the $K$ band by Dyck et al. (1998) led to an angular diameter of $7.2 \pm 0.5$ mas. This is in agreement, within the error bars, with our estimation given in Table 2. Further observations would be useful to assess whether there is a measurable dependence of the angular diameter with wavelength. Neither the interferometric measurements nor our LO indicate evidence of binarity. 
Table 2. Summary of results.

\begin{tabular}{|c|c|c|c|c|c|c|c|c|c|}
\hline (1) & (2) & (3) & (4) & (5) & (6) & (7) & (8) & (9) & (10) \\
\hline Source & $|V|$ & $V / V_{\mathrm{t}}-1$ & $\psi$ & PA & $\mathrm{CA}$ & SNR & Sep. (mas) & Br. Ratio & $\phi_{\mathrm{UD}}$ (mas) \\
\hline SAO 164567 & 0.6443 & $3 \%$ & & (74) & (11) & 14.3 & $2.0 \pm 0.1$ & $1.7 \pm 0.1$ & \\
\hline $30 \mathrm{Psc}$ & 0.2473 & $-44 \%$ & 20 & 122 & 69 & 46.1 & & & $6.78 \pm 0.07$ \\
\hline SAO 78119 & 0.5387 & $-3 \%$ & 2 & 129 & 41 & 52.7 & $13.1 \pm 1.1$ & $34.2 \pm 2.5$ & \\
\hline V349 Gem & 0.9462 & $-2 \%$ & 8 & 106 & 11 & 65.9 & & & $5.10 \pm 0.08$ \\
\hline SAO 78258 & 0.6307 & $2 \%$ & 1 & 45 & -50 & 9.4 & $47.3 \pm 1.5$ & $8.6 \pm 0.7$ & \\
\hline$A G+24788$ & 0.6910 & $3 \%$ & 6 & 75 & -13 & 16.9 & $28.8 \pm 0.7$ & $4.9 \pm 0.2$ & \\
\hline SAO 79251 & 0.7215 & $-1 \%$ & -1 & 85 & -15 & 20.2 & $26.9 \pm 1.1$ & $17.6 \pm 1.5$ & \\
\hline
\end{tabular}

\section{3. $S A O 78119, S A O 78258, A G+24788, S A O 79251$}

No previous indications of binarity for these stars are reported in the literature. SAO 78258 was listed in the Hipparcos catalogue as single star.

\subsection{V349 Gem}

No previous high-angular resolution measurements are listed for this carbon star in the literature. Epchtein et al. (1987) have classified it as having a temperature below $2500 \mathrm{~K}$, and a circumstellar dust shell of 400-1500 K. Our value for the angular diameter of this star seems to be consistent with its general properties. However, the spectral energy distribution of this source is poorly known, also considering its variability, and it is not possible to constrain significantly the effective temperature. Further photometric monitoring is desirable.

\subsection{Stars with negative binary detection}

Among the stars for which we did not detect any binarity, a few are known to have companions. After a close examination of the characteristics of the stars and a comparison with the circumstances and achieved performance of our observations, we conclude that there are no significant discrepancies (see Table 3 for a brief explanation of each non-detection). In the following, we provide a discussion of the individual cases which deserve special attention.

SAO 78168 was reported to be a double from by visual occultation (Zhitetski 1973). However, this observation was catalogued as doubtful in XZ80 catalog (Dunham \& Warren 1995) as the event was recorded to show gradual disappearance (Dunham \& Herald 2004). In addition, no binarity is reported in the Hipparcos catalogue. Finally, we note that the occultation trace of this star could be recorded at a very good $S N R$, permitting us to cover a dynamic range of almost five magnitudes from the primary.

SAO 79257 is a known subarcsecond binary, reported in the Washington double star (WDS J07181+2405) as well as in the Hipparcos (HIP 35344) catalogues. However, the entries are not entirely consistent for what concerns the position angles. WDS reports PA of $153^{\circ}$ and $132^{\circ}$ for epochs 1971 and 1991 respectively, with a separation of $0^{\prime} .4$ in both cases and a
Table 3. Summary of negative detection results.

\begin{tabular}{lrrrl}
\hline \hline \multicolumn{1}{c}{$(1)$} & $(2)$ & \multicolumn{1}{c}{$(3)$} & \multicolumn{1}{c}{$(4)$} & \multicolumn{1}{c}{$(5)$} \\
\multicolumn{1}{c}{ Source } & \multicolumn{1}{c}{$\psi$} & \multicolumn{1}{c}{ PA } & \multicolumn{1}{c}{ SNR } & \multicolumn{1}{c}{ Notes } \\
\hline SAO 164553 & -4 & 122 & 2.9 & Outside field of view \\
SAO 165578/B & 3 & 22 & 5.9 & Too faint \\
SAO 165578/C & & & & Outside field of view \\
SAO 78122 & 6 & 87 & 26.6 & Large separation \\
SAO 78168 & 0 & 73 & 78.9 & No details known \\
SAO 78197 & 12 & 110 & 31.8 & Outside field of view \\
SAO 79257 & 10 & 74 & 6.0 & Consistent with projection \\
\hline
\end{tabular}

magnitude difference of about unity. Hipparcos reports a PA of $158^{\circ}$ in 1991 , with a separation of 0.393. Given that the scan angle of our LO event $\left(74^{\circ}\right)$ was almost orthogonal to the PA reported above, the differences between Hipparcos and WDS are significant. We have used a binary star model to fit our occultation data of SAO 79257. The result was that the data are consistent with a binary having a separation of about 20 mas and a brightness ratio of about 2 mag (i.e., at the limit of the sensitivity permitted by the $S N R$ ). The resulting $\chi^{2}$ was improved by only $4 \%$ with respect to the case of a single star model, and we cannot claim a positive detection. Our derived projected separation can be reconciled with the true separation, if the LO scan direction was about $87^{\circ}$ from the PA of the binary. This would imply PA close to $161^{\circ}$, which is very close to the value measured by Hipparcos in 1991. We conclude that our data are not inconsistent with the presence of the known companion, and indicate that it would have to be significantly redder than the F5 primary. However, further conclusions are not possible given the uncertainties in the actual PA of the binary.

\section{Performance}

\subsection{Limiting magnitude}

By plotting the $S N R$ as a function of the magnitude of the occulted star, we can estimate an empirical relation for the limiting magnitude that can be achieved by observations with the two instruments, as shown in Figs. 1 and 2. 


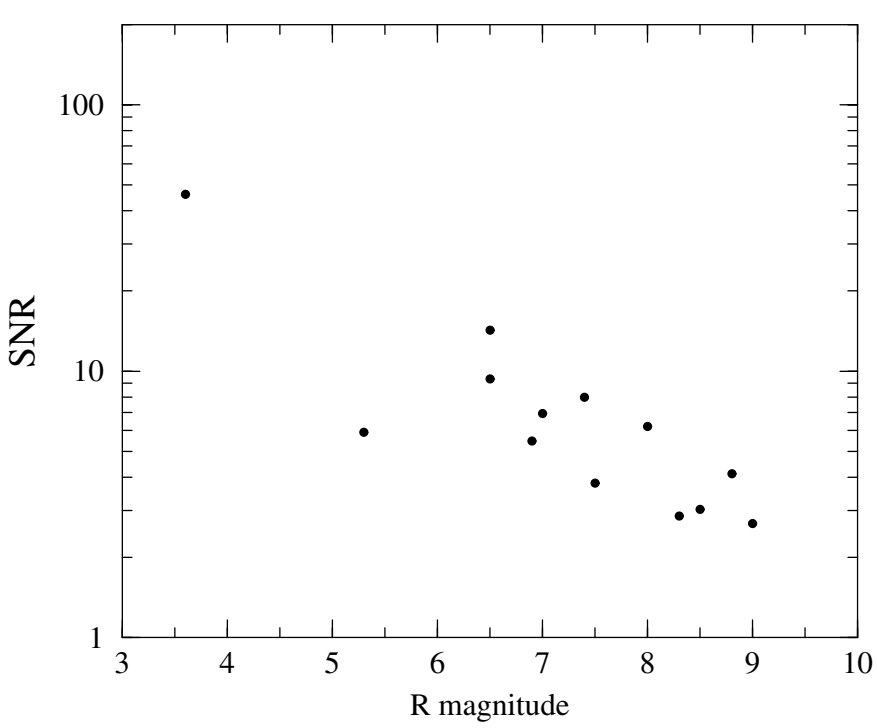

Fig. 1. Relation between $S N R$ and $R$ magnitude, for observations with the SBIG-ST8 instrument.

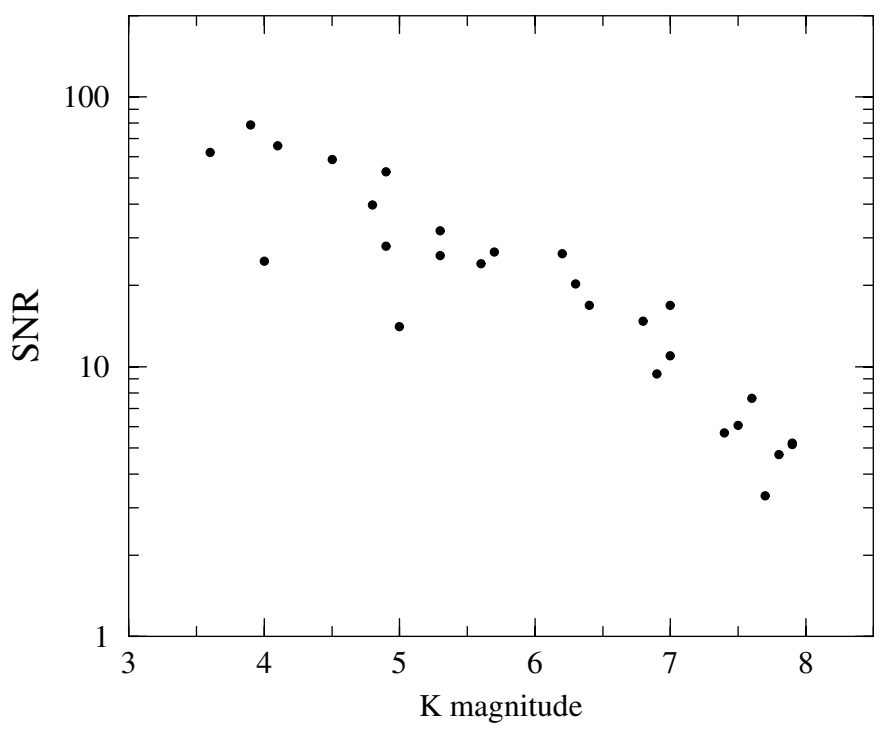

Fig. 2. Relation between $S N R$ and $K$ magnitude, for observations with the MAGIC instrument.

It can be noted that in both cases the data indicate a clear relationship between magnitude and $S N R$. For studies of binary stars, companions with a brightness ratio close to unity can be detected already when the $S N R$ is relatively small, in the range $1-3$. Figures 1 and 2 show that LO observations at the $1.5 \mathrm{~m}$ telescope can be used for investigations of binary systems down to magnitudes $R \approx 9$ and $K \approx 8.5$.

It is interesting to compare our result of Figs. 2 with 3 of Richichi et al. (1996b), which showed a similar plot for LO data obtained also with a $1.5 \mathrm{~m}$ telescope (TIRGO) in the $K$ band, but using a fast photometer. The IR array shows better $S N R$ for the range $K \approx 4-7 \mathrm{mag}$, probably thanks to the ability to reject more background signal and thus reduce significantly the photon noise in the data. Below $K \approx 7$ mag the advantage is less clear, due also to the scarcity and scatter of the data available for a comparison. One possible reason could be that LO events

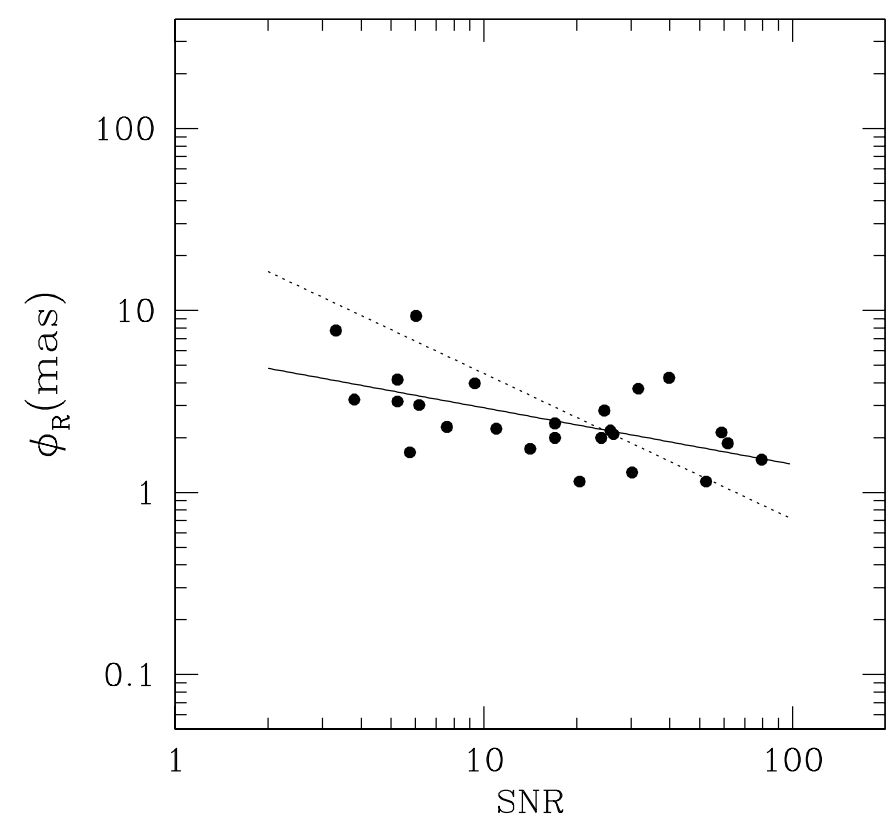

Fig. 3. Limiting resolution $\phi_{\mathrm{R}}$ for the unresolved sources in our sample, as a function of $S N R$. The solid line represents a linear fit, while the dotted line is the trend shown in Fig. 5 of Richichi et al. (1996b).

at TIRGO for such faint sources were recorded under conditions systematically better than average in terms of background (for example, at low lunar phases). Our Fig. 2 does not reach magnitudes brighter than $K \approx 3.5$, so no comparison can be drawn at the bright end.

One can also notice a much larger scatter in the figure based on the TIRGO data. This is probably due to the fact that the TIRGO sample include a much larger database of LO observations, collected over a wide range of lunar phases and background conditions, as well as with different settings of the photometer. Our data were instead recorded over three consecutive nights, and with essentially identical settings of subframe size and integration time.

\subsection{Limiting resolution}

We have also performed an analysis of the limiting angular resolution achieved in our observations, by adopting the same definition of resolution and the same approach described at Richichi et al. (1996b). In particular, the same code for the automatic estimation of the limiting angular resolution has been used. This quantity has been computed for 25 unresolved sources in our sample (this is less than the full size of the sample minus the resolved sources, because in a few cases the SNR was not sufficient to perform the computations), and is plotted as a function of $S N R$ in Fig. 3.

The figure shows, as expected, an improvement in the limiting angular resolution for increasing SNR. In particular, diameters below 2 mas are expected to be resolved for $S N R$ values approaching 100 . However, the slope of this function is significantly different from that of LO measurements obtained with a photometer. Figure 3 shows the linear approximation (by least-squares fits) of our data, and the same from Fig. 5 
of Richichi et al. (1996b) for the TIRGO telescope. It can be appreciated how the data obtained with CCDs and IR arrays, as in the present paper, provide a better performance in terms of limiting angular resolution in the low SNR regime. This is due to the better performance on faint sources, as already discussed in Sect. 4.1. However, the data obtained with the TIRGO photometer provide better angular resolution for $S N R$ values above $20-30$. This is probably due to the fact that in the bright source regime the advantages of arrays are less important, and the improved time sampling offered by photometers becomes important. Indeed, the sampling time achieved with our instruments (see Col. 5 of Table 1) is 2 to 4 times slower than what can be achieved by a fast photometer. In this respect, we hope to optimize some aspects of the electronic and acquisition software in the near future. In parallel, other improvements could be brought by the new generations of detectors currently being introduced and by the increasing allocating time availability in larger telescopes, as discussed in Appendix A.

\section{Conclusions}

We have described a routine program of lunar occultation observations started at the Spanish $1.5 \mathrm{~m}$ telescope of the Calar Alto observatory, using two relatively economical instruments for the visual and near-IR range. A total of 40 occultations were recorded, 13 in the visual and 27 in the nearinfreared, resulting in five binary detections and 2 diameter measurements. This being a small sample, it is hard to infer any statistical conclusions; however, it is interesting to note that the fraction of detected binaries (5/40, of which 4/40 represent new detections) is very similar to the value of $\approx 10 \%$ calculated for field stars by Evans et al. (1983) and Richichi et al. (1996b).

We have evaluated the performance of the two instruments in terms of signal-to-noise ratio achieved for LO observations, and we conclude that their use at the $1.5 \mathrm{~m}$ telescope makes it possible to carry out discoveries of binaries among field stars down to the limit of about 8-9 mag in the $R$ and $K$ bands. We have compared these observations with the database of nearinfrared lunar occultations recorded with a fast InSb photometer at the $1.5 \mathrm{~m}$ TIRGO telescope (Richichi et al. 1996b), and we conclude that the use of an array detector offers significantly better sensitivity.

Acknowledgements. This work was supported in part by the DGICYT Ministerio de Ciencia y Tecnología (Spain) under grant No. AYA2001-3092. O. Fors was supported by a fellowship from DGESIC Ministerio de Educación, Cultura i Deportes (Spain),
Ref. AP97 38107939. We thank the Observatorio Astronómico Nacional for the facilities and support made available at Calar Alto. We would like to express our gratitude to Christoph Flohr for making available his program SCAN. We thank David Dunham and Dave Herald for SAO 78168 information supplied in private communication. This research has made use of the USNOFS Image and Catalogue Archive operated by the United States Naval Observatory, Flagstaff Station (http://www.nofs.navy.mil/data/fchpix/). This project makes use of data products from the Two Micron All Sky Survey, which is a joint project of the University of Massachusetts and the Infrared Processing and Analysis Center/California Institute of Technology, funded by NASA and the NSF. This research has made use of the Simbad database, operated at CDS, Strasbourg (France).

\section{References}

Apogee Instruments Inc 2003, Alta Cameras Announcement (available at http://www.ccd.com/alta.html)

Dyck, H. M., van Belle, G. T., \& Thompson, R. R. 1998, AJ, 116, 981

Duflot, M., Figon, P., \& Meyssonnier, N. 1995, A\&AS, 114, 269

Dunham, D., \& Warren, W. H. Jr. 1995 (available at ADC/CDS http://cdsweb.u-strasbg.fr/viz-bin/ VizieR-2?-source=I/201)

Dunham, D., \& Herald, D. 2004, private communication

e2v Technologies 2002, e2v Technologies L3Vision[tm] high sensivity imaging $\mathrm{CCD}$ for low light applications (available at http://e2vtechnologies.com/introduction/ prod_l3vision_nojs.htm)

Epchtein, N., Le Bertre, T., Lepine, J. R. D., et al. 1987, A\&AS, 71, 39

Evans, D. S. 1983, Obs. Bull. (ISSH 0024-7057), 167, 73

Fors, O., Núñez, J., \& Richichi, A. 2001, A\&A, 378, 1100

Guglielmo, F., Epchtein, N., Arditti, F., et al. 1997, A\&AS, 122, 489

Herbst, T. M., Beckwith, S. V., Birk, C., et al. 1993, Proc. SPIE, 1946, 605

Mason, B. D., Martin, C., Hartkopf, W. I., et al. 1999, AJ, 117, 1890

Moore, J. H., \& Paddock, G. F. 1950, ApJ, 112, 48

Richichi, A. 1989, A\&A, 226, 366

Richichi, A. 1994, Very High Angular Resolution Imaging, IAU Symp., 158, 71

Richichi, A., Calamai, G., Leinert, Ch., et al. 1996, A\&A, 309, 163

Richichi, A., Baffa, C., Calamai, G., \& Lisi, F. 1996b, AJ, 112, 2786

Richichi, A., Calamai, G., \& Stecklum, B. 2002, A\&A, 382, 178

Richichi, A., \& Calamai, G. 2003, A\&A, 399, 275

Richichi, A. 2003, A\&A, 397, 1123

Rose, J. A. 1985, AJ, 90, 787

Sloan, G. C., \& Price, S. D. 1998, ApJS, 119, 141

Wang, S., Tang, Z., Jin, W., et al. 1998, Kinematika i Fizika Nebesnykh Tel, 14, 149

Zhitetski, A. 1977, IOTA Occultation Newsletter 1 (10), 110 


\section{Online Material}




\section{Appendix A: Improvements and future possibilities}

The technologies involved in both CCD and IR array manifacturing are under continuous improvement, thus producing detectors with better performance.

For what concerns CCDs, two important achievements have recently occurred. Firstly, subelectron readout noise has been achieved for the first time thanks to low-light chip technology (e2v Technologies 2002). This has been a major step forward for low signal applications such as LO. Secondly, on-board image memory has been recently implemented in commercial cameras (Apogee 2003), making it possible to record fast frame sequences without being limited by the data transfer interface throughput (USB, Ethernet, etc.). This is of crucial importance for LO observations in the visual, since it opens the possibility of recording occultations at millisecond rates on the basis of real subframe mode, as opposed to the drift-scanning technique which only records the flux on one pixel.

Catering to the needs of adaptative optics, new IR arrays with readout noise of very few electrons are presently being introduced. In addition, faster on-board image storage memory will allow next-generation arrays to increase the time sampling of the occultation to 1 or $2 \mathrm{~ms}$, yielding an improvement in limiting resolution.

As a result, a significant improvement in $S N R$ and resolution is to be expected in the near future. In particular, it is hoped that such technological achievements will be transferred to a wide range of detectors, and become available also to relatively low-budget programs such as the one here described. In particular, access to fast, sensitive detectors at affordable cost could be the key to promote LO observations not only at professional large observatories, but also at smaller facilities. In turn, this could partially overcome some of the intrinsic limitations of LO, such as the lack of repeated observations at various wavelengths, epochs and position angles.

In parallel to the improvements expected from detector developments, the trend in telescope availability also holds promises of increased LO performance in the near future. While LO have the advantage of providing an angular resolution which is not limited by the diffraction limit of the telescope, the technique is of course not insensitive to benefits of observing with large facilities. In particular for the case of binary stars, the increase in SNR achieved by moving to a large telescope is reflected directly in the range of brightness ratios of possible companions that can be explored, and also extends dramatically the number of stars that can be studied.

The extrapolation of LO observations to larger telescopes can be split into two diameter regimes. Telescopes in the 3-4 $\mathrm{m}$ class offer growing availability due to the increasing number of 8-10 m telescopes. The forthcoming 30-100 $\mathrm{m}$ facilities will accentuate this trend even more. By making use of flexible time allocation schemes, routine LO observations could be implemented with this class of telescope. Typically, a $3.5 \mathrm{~m}$ telescope would offer a limiting magnitude gain of about 1.5 units in $K$ with respect to the facility used in the current paper (Richichi 1994). Telescopes in the 8-10 m class could be used for special opportunities, and achieve a performance of the utmost quality. For example, Richichi (2003) has investigated the possibility to use LO at very large telescopes to perform detailed studies of stars with exoplanet candidates. In the years 2004 to 2008, up to 14 events could be observed from the largest observatories. 